\title{
Dinámicas de regeneración en las cooperativas multinacionales de Mondragón: la reproducción del modelo cooperativo en las filiales capitalistas
}

\author{
Ignacio Bretos Fernández \\ Anjel Errasti Amozarrain
}

\section{RESUMEN}

Tras dos décadas inmersas en dinámicas de internacionalización que han fortalecido su competitividad y capacidad de generación de empleo y riqueza pero que, a su vez, han cuestionado su naturaleza democrática tradicional, las cooperativas multinacionales de la División Industrial de Mondragón Corporación vienen asumiendo desde hace unos años un discurso centrado en fomentar la participación de los trabajadores en sus filiales capitalistas. Sin embargo, hasta ahora las iniciativas adoptadas en esta dirección son escasas y sus resultados no parecen excesivamente relevantes. El presente trabajo se centra en esta disociación, profundizando en la problemática y las posibles soluciones para extender y reproducir el modelo cooperativo en las filiales mercantiles. Analizamos el caso de Fagor Ederlan, focalizando nuestro estudio empírico en la "cooperativización" de su filial de Tafalla, así como investigaremos la casuística más relevante que se concentra en sus filiales extranjeras.

PALABRAS CLAVE: Cooperativa, Internacionalización, Mondragón, Regeneración, Democracia.

CLAVES ECONLIT: P13, J54, F23, M14, M12.

Cómo citar este artículo: BRETOS, I. \& ERRASTI, A. (2016): "Dinámicas de regeneración en las coo-

perativas multinacionales de Mondragón: la reproducción del modelo cooperativo en las filiales capitalistas", CIRIEC-España, Revista de Economía Pública, Social y Cooperativa, 86, 5-34.

Correspondencia: Ignacio Bretos Fernández, GEZKI - Instituto de Derecho Cooperativo y Economía Social, Universidad del País Vasco (UPV/EHU). Plaza Elhuyar, 2, 20018, Donostia-San Sebastián, España. E-mail: ignaciobretos@gmail.com; Anjel Errasti Amozarrain, Profesor Titular en Escuela Universitaria de Estudios Empresariales de Donostia-San Sebastián, Universidad del País Vasco (UPV/EHU). Plaza Oñati 1, Donostia-San Sebastián, 20018, España, y GEZKI. 


\section{EXPANDED ABSTRACT}

\section{Regeneration dynamics in Mondragon's multinational-cooperatives: the reproduction of the co-operative model at capitalist subsidiaries}

In the past few decades globalization, as a process that stimulates capitalism, has taken off at an unprecedented rate. One of the key dynamics defining this new globalization in the field of business organization is the spread of international expansion to almost all kinds of economic activity. It can be observed that many small and medium-sized enterprises have been forced to adopt global strategies in order to be competitive. Worker cooperatives have not remained untouched by this phenomenon, as evidenced by the emergence of numerous cooperative corporate groups that operate on an international scale with subsidiaries abroad. This globalized scenario has accentuated the degenerative tensions that cooperatives have always had to face in their efforts to uphold their traditional principles and values as they grow in a capitalist setting. This is in fact one of the great challenges facing cooperatives today: the conflict entailed by developing processes of internationalisation that are consistent with their nature. In this vein, as several authors note, currently a key line of research is to investigate how multinational cooperatives can replicate their policies and practices in their capitalist subsidiaries.

The Mondragon Group is a stand-out example of the way in which cooperatives have had to grow internationally to remain competitive. It is made up of about 250 cooperatives, and is the 10th biggest corporate group in Spain, with 74,060 employees all over the world. Since the 1990's several of its industrial cooperatives have undertaken internationalization processes that have entailed major increases in their output and in the volume of business that they do on international markets. In 2012 Mondragon's Industrial Division comprised 28 multinational cooperatives and 105 foreign subsidiaries, employing a total of 36,963 people (nearly 14,000 of them abroad). The total sales of the industrial division amounted to $€ 5.812$ million and $€ 4.004$ million corresponded to international sales, thus resulting in a ratio of international sales of $68.9 \%$.

This strategy has increased the competitiveness of these cooperatives and their ability to generate wealth and employment both in the Basque Country and abroad. It has also helped them to weather the current crisis. However, this model of growth based on setting up capitalist subsidiaries abroad calls into question their cooperative nature, as evidenced for instance by the fall in the ratio of cooperative employment. In recent years Mondragon has been promoting a discourse of regeneration based on the importance of encouraging worker participation at its capitalist subsidiaries. In fact, the 8th 


\section{DINÁMICAS DE REGENERACIÓN EN LAS COOPERATIVAS MULTINACIONALES DE MONDRAGÓN: LA REPRODUCCIÓN DEL MODELO COOPERATIVO EN LAS FILIALES CAPITALISTAS}

Mondragon Cooperative Congress (2003) approved a strategy of expansion based on the dissemination of cooperative values at subsidiaries by means of mechanisms for participation similar to those in place at the core cooperatives. In recent years some cooperatives have adopted a discourse based on the need for democratic regeneration at their organisations and on fostering the participation of the workers employed by their capitalist subsidiaries. However, to date few such initiatives have been implemented and results have been limited, especially among foreign subsidiaries. Strangely, the specialized literature has paid little attention to this issue.

This article aims to fill that gap. It sets out to investigate the tensions and opportunities entailed in setting up regeneration schemes at multinational cooperatives, focusing on the problems parent cooperatives face in reproducing democratic structures in their capitalist subsidiaries and on potential solutions for those problems. To this end, we conduct a case study of Fagor Ederlan. Founded in 1963, this organization is currently one of the biggest, most important multinational cooperatives at Mondragon, and one of the group's pioneers in expanding into international markets. Its model of internationalization is just like those used by the other large industrial cooperatives in the group: it has grown by acquiring and starting up capitalist firms, first in the domestic market and subsequently abroad. It has plants in strategic regions such as Eastern Europe, America and Asia. Moreover, it is at the cutting edge of development in regeneration processes consisting of implementing the cooperative model at its capitalist subsidiaries. Our analysis goes into greater depth than any that we have found in earlier papers, and focuses on a specific case of democratic regeneration: the transformation of the capitalist subsidiary Fagor Ederlan Tafalla - with over 900 employees - into a mixed cooperative, which is the biggest pioneering initiative of its kind taken to date by a Mondragon industrial cooperative.

The method used to investigate Fagor Ederlan is that of "contemporary case studies", and it is based on various techniques and drawn on various sources. 11 in-depth face-to-face interviews were held with staff from different areas of Fagor Ederlan. We also consulted a range of documentation provided by the company (sustainability reports, strategy plans, company articles, internal regulations, etc). Furthermore, we also contacted core organisations at Mondragon involved in regeneration processes at the group's cooperatives, such as the LANKI Institute at the University of Mondragon and the MIK (Mondragon Innovation \& Knowledge) research centre. Finally, we consulted external publications concerned with Fagor Ederlan and the Mondragon group, press releases and material from their own websites.

The limitations of the research reside in drawing broader conclusions for multinational cooperatives based on the analysis of a single case. Nonetheless, the findings obtained are significant both for academics and practitioners. Our findings suggest that regeneration is feasible at multinational cooperatives, either through the conversion of the capitalist subsidiaries into cooperatives or via the promotion of worker participation in such subsidiaries. Moreover, our investigation sheds new light by pointing out possible solutions that may be extrapolated to the democratic regeneration of foreign subsidiaries based on an analysis of the cooperativization of the domestic subsidiary Fagor Ederlan Tafalla. 
In this vein, the democratization of foreign subsidiaries can be tackled by turning them into "mixed cooperatives", in which the degree of control by the parent company is gradually decreased as worker members acquire experience in managing the subsidiary democratically and efficiently. To that end, preliminary processes of information, training and education in cooperative values for workers seem to be essential to establish the basis for future cooperativization schemes.

On the other hand, we have also found evidence of external obstacles and internal reluctance along the regeneration process. There are certainly major challenges and tensions involved in fostering such regeneration practices, especially at foreign subsidiaries. Evidence of external barriers has been found at Fagor Ederlan: they include several economic, legal and cultural barriers that hamper the implementation of the cooperative model at subsidiaries. Furthermore, our research goes further and suggests that there may well be internal reluctance and obstacles at parent companies which are even more important, such as the concentration of decision-making power and the wish to retain strategic activities and resources so as to maintain job stability of cooperative members at Basque plants.

In short, this paper makes further progress along the path laid out by previous works that analyze the contradictions in the internationalization of Mondragon and the regeneration schemes undertaken to tackle them. The data and assessments set out here may serve to encourage alternative management models and actions to enable firms that compete globally to grow sustainably on the basis of democratic, social values and principles. In this vein, our research provides helpful tools for practitioners to overcome the degenerative tensions that cooperatives face in the current globalized scenario. Finally, it also encourages taking actions at the institutional level in order to facilitate and adapt the legislations on cooperatives to these new realities and cooperativization initiatives.

KEY WORDS: Cooperative, Internationalisation, Mondragon, Regeneration, Democracy. 


\section{1.- Introducción}

La estrategia de internacionalización seguida desde los años 90 por las grandes cooperativas industriales de Mondragón Corporación Cooperativa (MCC) es un ejemplo de la adaptación del cooperativismo a la globalización desmarcándose en términos generales de la lógica de la deslocalización (Luzarraga, 2008; Luzarraga e Irizar, 2012; MacLeod y Reed, 2009), aunque igualmente evidencia diversos dilemas y contradicciones con su naturaleza tradicional (Errasti y Mendizábal, 2007), percibidos por muchos autores como una muestra de degeneración cooperativa. En los últimos años viene impulsándose un proceso de reflexión en MCC cuyo objeto es fomentar en sus cooperativas iniciativas de regeneración que acojan nuevamente sus principios y valores originarios, proponiendo entre otras líneas de actuación la implementación de una lógica cooperativista en las filiales capitalistas (Irizar, 2005; Bakaikoa et al, 2013). Sin embargo, se puede apreciar que las prácticas concretas adoptadas en esta dirección son relativamente escasas y sus resultados no todo lo relevantes que cabría esperar.

El objetivo del presente artículo es analizar esta disociación profundizando en la investigación de las dificultades y complejidades que implica para las cooperativas multinacionales de MCC el tratar de adecuar sus procesos de expansión a los principios y valores que les han caracterizado tradicionalmente, centrándonos concretamente en la problemática existente y las posibles soluciones para que las matrices cooperativas extiendan y reproduzcan su modelo democrático y participativo en sus filiales capitalistas vinculadas. Para ello, presentamos el estudio de caso sobre el Grupo Fagor Ederlan, una de las cooperativas multinacionales de Mondragón que más ha incidido en la potenciación de la participación y la democracia en el conjunto de su organización a través de la extensión del modelo cooperativo a sus filiales capitalistas, y que nos proporcionará un marco real para analizar los factores que generan las contradicciones existentes en dichas filiales y cómo se producen estos procesos degenerativos. Nos centraremos en el análisis del proceso de "cooperativización" en su filial de Tafalla y también consideraremos la problemática más relevante que se concentra en sus otras filiales capitalistas y las iniciativas que se han desarrollado para impulsar en ellas procesos de regeneración democrática. Este marco de análisis arrojará luz para proponer posibles soluciones a las dificultades que plantean desde Mondragón para impulsar estas iniciativas de regeneración, desvelando así posibles elementos subyacentes que pueden existir tras el hecho de que no se desarrollen más experiencias de este tipo.

Tal y como señalan Storey et al. (2014), la literatura sobre las empresas auto gestionadas por sus trabajadores ha estado dominada tradicionalmente por la "teoría de la degeneración". Nuestro trabajo parte de asumir y analizar estas dinámicas degenerativas en el ámbito de las cooperativas de Mondragón, pero situamos nuestras mayores contribuciones en las posibilidades que existen de impul- 
sar procesos de regeneración en las filiales capitalistas. En este sentido, analizamos con una transversalidad y profundidad que no encontramos en trabajos previos un proceso tan complejo como es la transformación de una filial capitalista en cooperativa; concretamente, el desarrollado en Fagor Ederlan Tafalla es uno de los proyectos de regeneración más potentes realizados en el ámbito industrial de Mondragón. Además, por su naturaleza, nos permitirá extraer ciertos aspectos transferibles a la cooperativización de las filiales capitalistas extranjeras, análisis que a su vez no se ha desarrollado en la literatura previa. Por último, es de subrayar que nuestro artículo trata de ir un paso más allá, identificando posibles reticencias internas en las matrices cooperativas que impiden que se fijen objetivos sólidos y políticas concisas para impulsar un mayor número de iniciativas dirigidas a transformar las filiales capitalistas.

Tras este apartado introductorio, se estructura nuestro trabajo de la siguiente manera. En el segundo apartado se ofrece una visión general de la internacionalización de las cooperativas de Mondragón, analizándose las dinámicas degenerativas en las que han incurrido y las principales iniciativas de regeneración que se están planteando. En el siguiente apartado se presenta el estudio de caso sobre Fagor Ederlan. En el cuarto apartado tratamos de recoger las principales dificultades y obstáculos existentes para extender el modelo cooperativo a las filiales, formulando a su vez posibles vías que nos aproximen a soluciones. Finalizaremos el artículo con un apartado conclusivo que también recoge las limitaciones de la investigación y posibles líneas futuras de estudio.

\section{2.- La internacionalización de las cooperativas de Mondragón. Una visión de conjunto}

La globalización ha experimentado desde los años 70-80 del pasado siglo una dinamización sin precedentes (Harvey, 2005). Entre las principales bases de acumulación capitalista que definen este nuevo estadio de la globalización podemos destacar el proceso de internacionalización de la producción y los mercados (Mendizábal, 1998, 2011), derivado de unas relaciones productivas y empresariales sustentadas en la "nueva división internacional del trabajo" (Fröbel et al, 1980) e impulsadas por la creciente competitividad internacional. Las medidas de liberalización económica y desregularización de los mercados, junto a las innovaciones tecnológicas acontecidas durante la década de los 90, han extendido el fenómeno de la internacionalización a prácticamente todo tipo de actividad económica, así como a la externalización de distintas fases de la cadena de valor de la misma empresa en diversos países (Mendizábal y Errasti, 2006). En definitiva, se constata que la globalización ha presionado a las pequeñas y medianas empresas a adoptar estrategias globales para ser competitivas (Acs y Preston, 1997; Gemser et al, 2004; Hedlund y Kverneland, 1985). 
Las cooperativas no han sido ajenas a estas dinámicas, tal y como demuestra el surgimiento de diversos grupos empresariales cooperativos que operan a nivel internacional (Côté, 2001; Chaves et al, 2003; Barea et al, 1999) en diferentes sectores de actividad como el industrial (por ejemplo, Metsa Group), el agrícola (Zen-Noh Group), el bancario (Crédit Agricole Group) o el de distribución (Rewe Group). Se pone así de manifiesto uno de los grandes retos contemporáneos del cooperativismo: la problemática existente en estas organizaciones para desarrollar procesos de internacionalización coherentes con su naturaleza, y sustentados por tanto en la reproducción del modelo cooperativo en sus filiales vinculadas (Radrigán y Barría, 2007; Flecha y Ngai, 2014), desvelándose el desafío histórico que la globalización ha presentado para que las cooperativas se desarrollen de acuerdo a sus principios y valores tradicionales (Spear, 2001; McMurtry y Reed, 2009; Zamagni y Zamagni, 2010). Sin embargo, algunas de estas cooperativas están haciendo frente a estas dinámicas degenerativas mediante iniciativas de regeneración democrática, como son los casos destacables de John Lewis Partnership en el área de distribución o de Mondragón Corporación especialmente en el ámbito industrial.

\subsection{Principales características y resultados de la internacionalización en Mondragón}

Desde mediados de los años 90 varias cooperativas industriales del conglomerado mondragonés, inmersas en sectores productivos globales muy dinámicos y competitivos, han seguido procesos de internacionalización que han implicado el crecimiento progresivo de su tejido productivo y volumen de negocio en los mercados internacionales, hasta el punto de que es en el exterior donde se concentra buena parte de su actividad productiva y comercial actualmente. Si bien ya existían previamente algunas actividades internacionales, encontramos el origen de esta orientación en el año 1993, cuando en el IV Congreso Cooperativo de Mondragón se establece la internacionalización como uno de los objetivos básicos, y posteriormente se aprueba el primer Plan Estratégico Corporativo de Internacionalización (PECI) para el periodo 1994-1996 (Urdangarín, 1999). Esta estrategia de internacionalización, cuyo objetivo último es mantener la competitividad para salvaguardar el empleo en las matrices, ha significado la transformación de muchas de estas cooperativas en multinacionales, al adoptar modelos clásicos de inversión directa en el extranjero (Bakaikoa et al, 2004) con prácticas además en algunos casos ciertamente similares a las de otras empresas multinacionales convencionales (Mendizábal et al, 2005; Errasti, 2013b).

De esta manera, entre 1991 y 2001 , el ratio de ventas internacionales sobre el total de ventas de las cooperativas industriales de Mondragón pasó del 25\% al 50,9\%. Durante el periodo 1999-2009, la división industrial de Mondragón generó 15.926 nuevos puestos de trabajo, de los que 12.715 fueron creados en el exterior. A finales del año 2012, el grupo industrial de MCC empleaba a un total de 36.963 personas. Esta división contaba ya con 28 cooperativas globales, de las que dependían 105 filiales extranjeras que daban empleo a un total de casi 14.000 personas, suponiendo alrededor del $40 \%$ del empleo industrial en Mondragón y el 17,5\% del empleo total de la Corporación. Así, el ratio de empleo cooperativo en las matrices se situaba en el $85 \%$, pero considerando el conjunto del grupo 
industrial, esto es, incluyendo las filiales, este ratio descendía al 45\% respecto al total del empleo, siendo por tanto mayoritario el empleo no cooperativo. Las ventas totales de la división industrial de Mondragón ascendieron en este año a 5.812 millones de euros, correspondiendo 4.004 millones a las ventas internacionales, resultando así un ratio de ventas internacionales sobre el total de ventas del 68,9\% (Mondragón, 2012a).

El procedimiento de internacionalización de estas cooperativas se ha basado en el establecimiento, mediante la adquisición o nueva creación (greenfield investment), de una periferia de filiales capitalistas alrededor de los núcleos cooperativos (Larrañaga, 2006: 230), donde sus trabajadores cuentan con unas condiciones socio-laborales muy alejadas de las ostentadas por los socios de las matrices (Defourny, 1999). Dichas filiales deben desarrollarse en torno a un modelo fuertemente jerarquizado, bajo estrechas relaciones de dependencia respecto a sus matrices, debido a que son éstas las que concentran el poder de decisión sobre las cuestiones estratégicas, y por tanto retienen la mayor capacidad de I+D, inversión, recursos estratégicos, actividades de mayor valor añadido, etc. (Errasti et al, 2003). Concretamente, como es ya conocido, la totalidad de las filiales extranjeras son sociedades anónimas (Irizar y MacLeod, 2008), siendo todos sus trabajadores asalariados contratados por cuenta ajena (Clamp, 2000).

De todas formas, debemos poner en relieve la vital diferencia entre el modelo de internacionalización de las multinacionales capitalistas y el de las cooperativas de Mondragón, que no es otra que el compromiso con el empleo local. Mientras que las estrategias expansionistas de las multinacionales capitalistas, de naturaleza activa o agresiva, se suelen basar en la deslocalización, cuyo carácter sustitutivo conlleva la destrucción de empleo, el modelo de internacionalización de las cooperativas de MCC, de naturaleza reactiva o defensiva, se basa en la "multilocalización", esto es, una estrategia expansionista y creativa en lugar de destructiva ya que la nueva actividad industrial abierta en el extranjero no implica el cierre de ninguna actividad preexistente. De esta manera se logra la competitividad necesaria para cumplir con el compromiso social de mantener el empleo cooperativo en el territorio local y crear empleo en términos generales en el exterior (Luzarraga et al, 2007; Luzarraga, 2008; Luzarraga e Irizar, 2012; Redondo et al, 2011; Arando et al, 2010).

En este sentido, Luzarraga et al (2007) señalaban, tomando como referencia el periodo 19962006, que el empleo en las cooperativas globales (las que disponen de filiales en el exterior) creció más que el empleo en las cooperativas locales (las que no cuentan con filiales extranjeras), existiendo en las primeras un crecimiento neto superior en los puestos de trabajo en los tres entornos: en las cooperativas matrices, en el entorno local y a escala internacional. Así mismo, destacaban que la pérdida de representatividad y control democrático de las cooperativas globales, en términos cuantitativos, no se había debido a la menor representatividad de los socios en las cooperativas matrices, cuya evolución había sido positiva en el periodo 1999-2006 (siendo además superior en las cooperativas globales), sino que se debió a que no se había reproducido la fórmula cooperativa en ninguna de las filiales extranjeras, lo que supuso una reducción de la representatividad de los socios sobre el total de trabajadores. 
Posteriormente, Luzarraga e Irizar (2012), demostraban tomando como referencia un periodo temporal más amplio (hasta el año 2009), que la reducción de empleo neto producida por la crisis económica iniciada en 2008 había sido mucho menor en las cooperativas globales que en las locales y que incluso en las primeras, había existido un aumento positivo del empleo cooperativo en sus matrices, por el descenso negativo en las segundas. De forma global, al igual que sucedió en crisis anteriores (Ellerman, 1984; Bradley y Gelb, 1982) se puede afirmar que en estos últimos años las cooperativas de MCC, en términos generales, están soportando satisfactoriamente la difícil coyuntura económica que venimos atravesando desde 2008 en comparación con las empresas convencionales (Roelants et al, 2012; Basterretxea y Albizu, 2009; Arando et al, 2010), gracias en buena parte a la distensión que les otorga su actividad internacional y al uso de mecanismos de solidaridad interna e intercooperación (Elortza et al. 2012). Sin embargo, también encontramos claras excepciones como la de Fagor Electrodomésticos. Durante varios años antes de su quiebra en 2013, el modelo de internacionalización desarrollado por esta cooperativa no se tradujo en un mantenimiento del empleo en las plantas vascas (Errasti y Mendizábal, 2007).

\subsection{Dinámicas degenerativas e iniciativas de regeneración}

Tal y como hemos constatado esta estrategia de "multilocalización", en términos generales, ha provisto de un gran éxito empresarial y de buenos resultados económicos a estas cooperativas y, además, se ha logrado con diferencias significativas respecto a las prácticas de deslocalización de las multinacionales capitalistas, ya que se ha creado empleo en el exterior mientras se mantenía y generaba empleo societario en las plantas matrices (Mondragón, 2012b). Sin embargo, también se manifiesta que estos procesos de internacionalización han generado fuertes tensiones y contradicciones con el modelo cooperativo, resultando evidente que, después de varias décadas, la lógica de mercado y competitividad global ha predominado en estas cooperativas sobre sus valores originarios de participación y democracia, cuestión retratada en el hecho de que actualmente muchas de las cooperativas industriales de MCC cuentan ya con más trabajadores asalariados en sus filiales capitalistas extranjeras que socios trabajadores en sus matrices cooperativas (Errasti, 2013a). Por ejemplo, en el caso de Fagor Electrodomésticos, antes de su caída, apenas un 30\% de los trabajadores del grupo eran socios (Mendizábal et al, 2005). Este conflicto derivado de la reducción del ratio de empleo cooperativo también es patente en otras áreas de Mondragón como la de distribución (Bakaikoa et al, 2013; Storey et al, 2014), donde Eroski pasó de tener un ratio de empleo cooperativo del 34,8\% en el año 2000 a tan solo el $16 \%$ en 20081 .

De esta manera, la teoría de la degeneración cooperativa, desarrollada por multitud de autores como Potter (1891), Webb y Webb (1920), Meister (1974, 1984), Ben-ner (1984) o Miyazaki (1984), la cual predice que las cooperativas están destinadas ineludiblemente al fracaso o a la degeneración

1.- Si tenemos en cuenta los trabajadores con una propiedad parcial en las filiales GESPA, este ratio en 2008 aumenta al $28 \%$ (Bakaikoa et al, 2013).

CIRIEC-España, Revista de Economía Pública, Social y Cooperativa 
democrática en formas capitalistas ${ }^{2}$, queda reflejada en algunas de las cooperativas multinacionales de MCC, tal y como sugieren los trabajos de Errasti, et al (2003), Bakaikoa, et al (2004), Luzarraga et al (2007), Huet (2000) o Cheney (1999), siendo la internacionalización un elemento clave en dicho proceso. Aludiendo a Cornforth (1988), este modelo de expansión ha implicado la degeneración constitucional, al contar con filiales que funcionan bajo fórmulas capitalistas, la degeneración de objetivos, al atender más a fines capitalistas que cooperativos, y la degeneración de la organización, al concentrarse el poder de decisión en una élite conformada por las altas instancias de las matrices.

Sin embargo, en estos últimos años, algunas de las cooperativas de MCC están cuestionándose su rumbo y tratando de volver a acoger en mayor o menor grado sus principios y valores originarios, desarrollando procesos de regeneración que se centran en fomentar la democracia y participación en sus organizaciones (Azkarraga et al, 2012). Este proceso de reflexión tiene su origen en el VIII Congreso Cooperativo de MCC celebrado en mayo de 2003, cuando se aprueba una estrategia de expansión social (Irizar, 2005) consistente en la difusión de los valores cooperativos en las filiales mediante el desarrollo de mecanismos de participación similares a los disponibles para los miembros de sus cooperativas (Flecha y Ngai, 2014). A través del Instituto Lanki de Mondragon Unibertsitatea se está tratando de inculcar la filosofía del cooperativismo en las filiales extranjeras mediante proyectos de fomento de los principios y valores cooperativos tanto a nivel de los órganos de la Corporación como de las propias cooperativas del grupo (Altuna, 2008; Azkarraga, 2007; Bakaikoa et al, 2013). De esta forma, siguiendo la línea de autores como Batstone (1983), Cornforth (1995), Cornforth et al. (1988) o Estrin y Jones (1992), que señalan que la degeneración en las cooperativas no es inevitable y que en cualquier caso pueden desarrollar procesos de regeneración que les devuelvan su sentido democrático y participativo, en Mondragón se pueden observar políticas y prácticas orientadas a dicha finalidad.

Podemos diferenciar dos grandes líneas de actuación: la primera y más directa es la "cooperativización" de la filial, existiendo fundamentalmente dos mecanismos. Por un lado, mediante la figura de la "cooperativa mixta"3, esto es, la filial capitalista se transforma en una cooperativa en la que una parte de la propiedad pertenece a los socios de la filial y otra parte generalmente a la matriz e incluso a terceros como Mondragón Inversiones. Por otro lado, existen casos en los que un número significativo de trabajadores de la filial capitalista pasan a ser socios de la matriz cooperativa, bajo la figura del socio en comisión de servicios ${ }^{4}$, aun manteniéndose la forma jurídica de dicha filial. Hasta ahora, este modelo de cooperativización se ha implantado exclusivamente a nivel doméstico. Es de destacar que estos procesos de cooperativización no son una práctica nueva en MCC y, ya en 2004, alrededor de un $15 \%$ del empleo de la división industrial provenía de estos modelos de transformación

2.- Podemos encontrar una revisión más amplia y detallada sobre las teorías de la degeneración cooperativa en el trabajo de Cornforth (1995).

3.- Las "cooperativas mixtas" son aquellas en las que existen socios minoritarios cuyo derecho de voto en la Asamblea General se podrá determinar, de modo exclusivo o preferente, en función del capital aportado.

4.- Éstos son aquellos socios que mantienen un vínculo societario con la cooperativa y prestan su trabajo en una entidad con la que la cooperativa coopera o participa. 
(Tulankide, 2004). La segunda línea de actuación se basa en la implementación del Modelo de Gestión Corporativo de Mondragón en las filiales capitalistas a través del modelo de "Desarrollo Societario" (Mondragón, 2013: 40-41), consistente en una serie de indicadores y de mínimos relativos a la gestión y participación de los trabajadores en las filiales capitalistas que trata de acercar su modelo de gestión al modelo cooperativo de sus matrices. Este modelo no implica la transformación de la filial en cooperativa ni la conversión de sus trabajadores en socios y está diseñado especialmente para las filiales extranjeras.

En este marco, encontramos trabajos como el de Luzarraga e Irizar (2012), Bakaikoa et al. (2013), Flecha y Ngai (2014) o Storey et al. (2014) que analizan experiencias de regeneración en cooperativas de Mondragón. Sin embargo, es constatable que estas experiencias son relativamente escasas y sus resultados no son excesivamente relevantes. En comparación con el total de filiales capitalistas de MCC que existen a nivel nacional, el modelo de la cooperativa mixta se ha implantado en un número reducido de ellas. Por otro lado, las experiencias de regeneración desarrolladas a través de la reproducción de la lógica cooperativa en las filiales extranjeras inciden en cuestiones como la mejora en las condiciones laborales de los trabajadores, las políticas de comunicación, sensibilización e información, o la inter-cooperación, pero ninguna constituye una experiencia de pleno éxito, entendiéndose por ello que la filial extranjera haya integrado en su funcionamiento la totalidad de los principios cooperativos. Dichas investigaciones revelan que no se ha avanzado significativamente en el fomento de la participación de los trabajadores de las filiales extranjeras, especialmente en lo referente a la propiedad y resultados y, en cuanto a la gestión, la participación se centra más bien en el área de trabajo y no tanto en el ámbito de decisión de los órganos de gobierno.

\section{3.- Estudio de caso: El Grupo Fagor Ederlan y el proceso de "cooperativización" de su filial de Tafalla}

\subsection{Metodología}

La metodología empleada para la investigación sobre Fagor Ederlan se ajusta al "estudio de casos contemporáneos" (Yin, 2003), que nos permitirá conocer en profundidad un proceso tan complejo como es la transformación de una sociedad anónima en cooperativa y nos servirá para analizar las contradicciones existentes en términos de democracia y participación en las filiales. Aun teniendo en cuenta las limitaciones motivadas por el análisis de un solo caso a la hora de extraer conclusiones generales para el conjunto de las cooperativas industriales de Mondragón, debemos destacar las fuertes potencialidades que residen en la selección de este caso concreto para aproximarnos fehacientemente al objetivo de nuestro estudio. Por un lado, Fagor Ederlan es una de las cooperativas industriales de 
mayor tamaño e importancia de Mondragón, así como una de las entidades punteras en el desarrollo de procesos de integración del modelo cooperativo en sus filiales vinculadas. Por otro lado, la cooperativización de Fagor Ederlan Tafalla, su filial de mayor tamaño, es uno de los proyectos más potentes desarrollados en este marco por cooperativas de Mondragón y, además, por sus características, nos permitirá identificar elementos extrapolables a la regeneración de las filiales extranjeras de cualquier cooperativa industrial.

Para la realización de esta investigación nos hemos servido de diferentes técnicas y nutrido de diversas fuentes. Por un lado, hemos realizado 11 entrevistas con personas clave que han participado en el proceso de cooperativización de la filial desde perspectivas y posiciones diferentes, incluso contrapuestas (7 entrevistas presenciales en profundidad y 4 telefónicas). Las presenciales se han realizado con (1) el Coordinador del Proyecto de Cooperativización de Tafalla, quien fue anteriormente Director de Recursos Humanos de Fagor Ederlan, (2) un Directivo de Fagor Ederlan que asumió el cargo en Tafalla para desarrollar el proyecto de cooperativización, (3) el Presidente de Fagor Ederlan Tafalla, que anteriormente había sido Presidente del Comité de Empresa de la filial, (4) el Jefe de Personal de la filial de Tafalla, cargo en el que se ha mantenido tras el proceso de cooperativización, (5) un miembro del Sindicato Comisiones Obreras Navarra relacionado con el proceso de cooperativización de Tafalla, (6) un trabajador de la filial que pasó a ser socio cuando ésta se transformó en cooperativa, y (7) un trabajador que se mantuvo como asalariado cuando la filial pasó a ser cooperativa. Por su parte, se han realizado dos entrevistas telefónicas $(8,9)$ con trabajadores que pasaron a ser socios y otras dos $(10,11)$ con trabajadores que se mantuvieron como asalariados.

Por otro lado, nos hemos servido de diversa documentación proporcionada por la empresa (Informes de Sostenibilidad, Planes Estratégicos, Estatutos Sociales, Reglamento Interno...), destacando un documento interno de trabajo en el que, a través de 127 entrevistas individuales con trabajadores de distintos colectivos de la filial, se realiza una investigación acerca del propio proceso de cooperativización y de sus resultados en términos de participación, involucración e integración de los trabajadores en el nuevo modelo cooperativo que asume la filial tras dicho proceso. Por último, es de destacar que se ha contactado con organizaciones del seno de Mondragón involucradas en procesos de regeneración de las cooperativas del Grupo, como el Instituto LANKI o el centro de investigación MIK (Mondragon Innovation \& Knowledge). Así, accedimos a un trabajo de MIK en el que se analiza la experiencia de los representantes sindicales en Fagor Ederlan Tafalla en torno a la participación de los trabajadores tras el proceso de cooperativización. Complementariamente, hemos recurrido a publicaciones relacionadas con el Grupo Fagor Ederlan y a material recogido en su página web.

\subsection{Visión General del Grupo Fagor Ederlan y de la filial de Tafalla}

Fagor Ederlan es una cooperativa de MCC dedicada actualmente al diseño, desarrollo y fabricación de componentes metálicos de Chassis (freno-suspensión) y Powertrain (motor-transmisión). Comenzando su expansión internacional en 1998, en el año 2007 se consolida el Grupo Fagor Ederlan, 
liderado por Fagor Ederlan S. Coop. y configurado en la actualidad, además de por sus 8 plantas guipuzcoanas, por sus sociedades participadas Fagor Ederlan Tafalla S. Coop., Fagor Ederlan Borja S.A. y Victorio Luzuriaga Usurbil en territorio nacional, y Fagor Ederlan Brasileira (Brasil), Fagor Ederlan Slovensko (Eslovaquia) y Fagor Ederlan Auto Parts Kunshan (China) en el extranjero ${ }^{5}$. Dispone de un Centro Tecnológico, EDERTEK S. Coop., en Mondragón y cuenta con 2 alianzas productivas en EEUU y Corea, y ocho oficinas comerciales en Europa, Asia y Norte América. En 2014, sus ventas ascendieron a 650 millones de euros (el 70\% fueron ventas internacionales). Realizaron inversiones valoradas conjuntamente en 43 millones de euros y el número total de la plantilla se situó en 3.600 personas.

Respecto a la filial de Tafalla, es de destacar que el Grupo Fagor adquiere en 1990 Victorio Luzuriaga S.A. y en 1995 entra en el accionariado Fagor Ederlan y MCC. En 1997 se divide la sociedad en Victorio Luzuriaga Usurbil y Victorio Luzuriaga Tafalla. La participación en el capital social de Victorio Luzuriaga Tafalla S.A. se dividió en partes iguales entre MCC, Grupo Fagor y Fagor Ederlan. Con el objetivo de lograr un mayor control sobre la filial, en 2005, Fagor Ederlan adquiere el $33 \%$ que tenía en propiedad el Grupo Fagor, quedando por tanto el capital repartido entre Fagor Ederlan (2/3) y MCC (1/3). En 2008 se transforma Victorio Luzuriaga Tafalla S.A. en la cooperativa mixta Fagor Ederlan Tafalla S. Coop., quedando el capital repartido en aquel momento entre sus socios (12\%), Fagor Ederlan S. Coop. (60\%) y MCC (28\%).

\section{Cuadro 1. Evolución de la propiedad de Victorio Luzuriaga Tafalla}

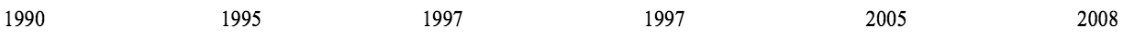

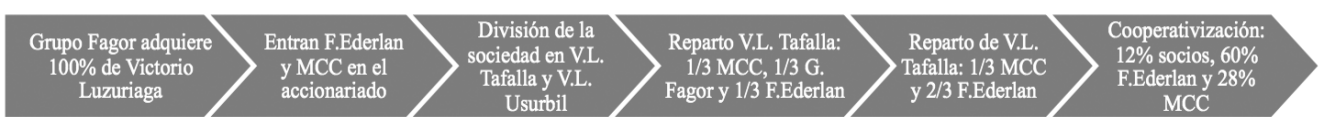

FUENTE: elaboración propia.

Fagor Ederlan Tafalla S. Coop. se dedica actualmente a la fundición de bloques y culatas para motores, estando integrada en la unidad de negocio de Powertrain. En 2014 contaba con una plantilla de 688 personas (485 socios, 52 trabajadores asalariados fijos y 151 eventuales). Sus ventas internacionales representaron el 65\% del total y sus inversiones para el periodo 2010-2014 ascendieron a casi 60 millones de euros.

5.- Próximamente contará con dos nuevas plantas productivas (sumando así un total de 18), nacidas de la firma de sendas joint-ventures en China, con el Grupo Infun, y en México con el Grupo Industrial Saltillo. 


\subsection{Claves del Proceso de Cooperativización de Victorio Luzuriaga Tafalla}

En el Plan Estratégico 2005-2008 de Fagor Ederlan se recogió como objetivo el desarrollo del empleo cooperativo a través de la cooperativización de sus filiales participadas. Ello se reflejaría en los proyectos de cooperativización desarrollados hasta ahora en tres filiales; Bergara, Usurbil y Tafalla. Cabe señalar que los procesos de cooperativización de las filiales Victorio Luzuriaga Usurbil y FIT Automoción Bergara son muy diferentes al de Tafalla, ya que en éstas no se ha transformado societariamente la filial sino que sus trabajadores se han integrado como socios en comisión de servicios de Fagor Ederlan S. Coop., pasando a ser plantas productivas de la matriz ${ }^{6}$. Respecto al caso concreto de la filial de Tafalla, en 2006 se empieza a trabajar desde Fagor Ederlan en su proyecto de cooperativización. Además de los compromisos fijados en el Plan Estratégico, también existieron razones de carácter estratégico y económico. En esos momentos, la filial de Tafalla requería de una fuerte inversión económica en los medios productivos y modelos organizativos, y desde Fagor Ederlan no se estaba dispuesto a realizar ese esfuerzo en una filial que funcionaba bajo una fórmula societaria capitalista.

De esta manera, se comienza por formar el "Equipo del Proyecto de Cooperativización", que optó por un modelo de cooperativización bajo la figura de la cooperativa mixta, participada por los futuros socios trabajadores de Fagor Ederlan Tafalla, por MCC y por Fagor Ederlan. Las razones para elegir este modelo de cooperativización y no uno más simple y directo como el de Bergara o Usurbil, son varias. Por una parte, existía una gran dificultad de reproducir ese mismo modelo de cooperativización en una región como Tafalla, alejada geográficamente de la matriz y donde la cultura cooperativa no está tan interiorizada como en el territorio guipuzcoano. Por otra parte, era necesaria la participación de MCC y Fagor Ederlan debido a que el desembolso económico necesario para hacerse con la propiedad total de la filial era inalcanzable para los futuros socios ${ }^{7}$. De todas formas, se estableció como objetivo a largo plazo que la filial de Tafalla termine siendo propiedad exclusiva de sus socios.

Una vez definido el proyecto, se pasa a estudiar su viabilidad, tanto legal como social y económica. En el plano legal, en un primer momento, la legislación navarra sobre cooperativas no contemplaba la posibilidad de desarrollar un proyecto de estas características. Este obstáculo se salvó ya que, gracias al trabajo de MCC y Fagor Ederlan, en noviembre de 2006 se aprueba la nueva Ley de Cooperativas navarra, que sí permitía desplegar el proyecto diseñado para la filial de Tafalla. En el plano económico, se realizó un estudio de la viabilidad económica de la planta, contemplando las necesidades requeridas de inversión que se iban a asumir desde la matriz, así como un análisis de negocio que determinase la rentabilidad de la planta a medio y largo plazo. La viabilidad social hacía

6.- Mientras que en el caso de FIT Bergara este proceso ya culminó y actualmente es considerada una de las 8 plantas productivas de Fagor Ederlan S. Coop., en el caso de Victorio Luzuriaga Usurbil se prevé finalizar el proceso en 2016.

7.- Para la adquisición inicial en 2008 del $12 \%$ del capital de la filial, cada uno de los socios tuvo que realizar un desembolso de 13.000 euros. 
referencia a la prospección de la receptividad del proyecto por parte de los trabajadores, que se estudió a través de multitud de entrevistas con ellos.

Posteriormente, tras un trabajo previo de coordinación y consenso con diversos actores involucrados (Dirección y Comité de Empresa de la filial, Consejo Rector de la matriz, INEM, Inspección de Trabajo, Gobierno de Navarra y Caja Laboral), se pasó a presentar el proyecto a los trabajadores de la filial, ofreciéndolo como un proyecto socio-empresarial indivisible: las inversiones en la planta estaban ligadas a su transformación en cooperativa. En este sentido, desde el Consejo Rector de Fagor Ederlan S. Coop., se fijó que para llevar adelante el proyecto, más de la mitad de los 750 trabajadores fijos y relevistas que había en ese momento debían convertirse en socios. Tras intensos procesos de comunicación y formación en los que se desarrollaron charlas informativas, entrevistas, encuestas no vinculantes, procesos de formación y educación cooperativa para los futuros socios, el 5 de enero de 2008 se transformó la empresa mercantil Victorio Luzuriaga Tafalla S.A. en la cooperativa mixta Fagor Ederlan Tafalla S. Coop.

\section{Cuadro 2. Fases del Proceso de Cooperativización en Fagor Ederlan Tafalla}

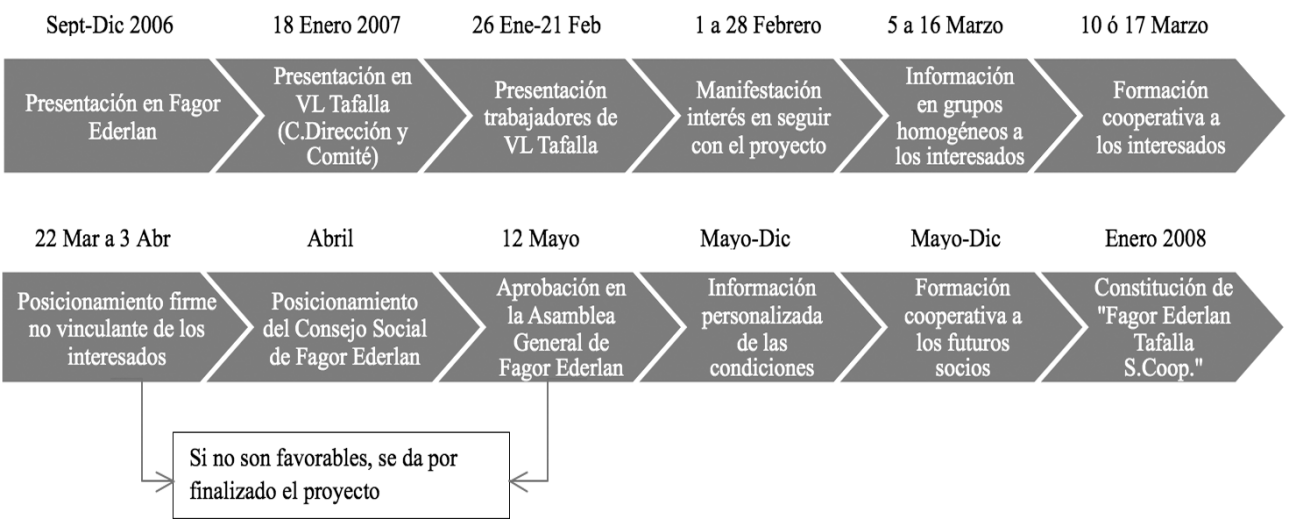

FUENTE: Fagor Ederlan. 


\subsection{Análisis de la democracia y participación en Fagor Ederlan Tafalla}

\section{Órganos de Gobierno de Fagor Ederlan Tafalla}

Dada la singularidad de la figura de la cooperativa mixta, según los Estatutos de Fagor Ederlan Tafalla, la Asamblea General no se rige por la norma habitual "una persona, un voto" para sus socios sino que existe mayoría de voto para Fagor Ederlan y MCC. En cualquier caso, nunca se ha utilizado este poder de decisión mayoritario por parte de Fagor Ederlan y MCC, quienes participan en las Asambleas Generales con un voto cada uno, al igual que los restantes 350 de los socios de Fagor Ederlan Tafalla. Por tanto, para cuestiones muy importantes como bajadas salariales, ampliaciones de capital, inversiones, etc., siempre se ha buscado un consenso previo, dependiendo las decisiones finales exclusivamente de sus socios trabajadores.

Respecto al Consejo Rector, es de señalar que, en lugar de reflejar una representación de los socios de la filial proporcional a su participación del 12\% en la propiedad, se configuró con 5 miembros que eran socios trabajadores de Tafalla y 7 miembros que pertenecían a MCC y Fagor Ederlan. De esta forma, las decisiones más importantes, que requieren según Estatutos las 2/3 partes de los votos, no podían ser aprobadas exclusivamente por la mayoría de MCC y Fagor Ederlan. Así mismo, se constituyó la "Comisión Delegada", órgano formado exclusivamente por los representantes de los socios trabajadores en el Consejo Rector y el Presidente de dicho órgano, y con competencias para decidir autónomamente sobre las cuestiones más operativas que surgen en el día a día de la filial. Por tanto, en relación a la propiedad de la filial que tienen sus socios trabajadores, éstos han ostentado una clara sobrerrepresentación en sus estructuras democráticas, gozando de un elevado grado de autonomía respecto a sus socios colaboradores y accionistas, Fagor Ederlan y MCC.

\section{Mecanismos de Profundización de la Participación en Fagor Ederlan Tafalla}

Tras su cooperativización, Fagor Ederlan Tafalla reprodujo en su organización el Modelo de Gestión de su matriz y, por tanto, el Sistema de Participación que integra. Dicho sistema se define como "la contribución, tomando parte activa, en la definición, ejecución, evaluación, coordinación y mejora en todos los procesos y ámbitos de actividad que desarrolla la empresa". 


\section{Cuadro 3. Sistema de Participación de Fagor Ederlan S. Coop.}

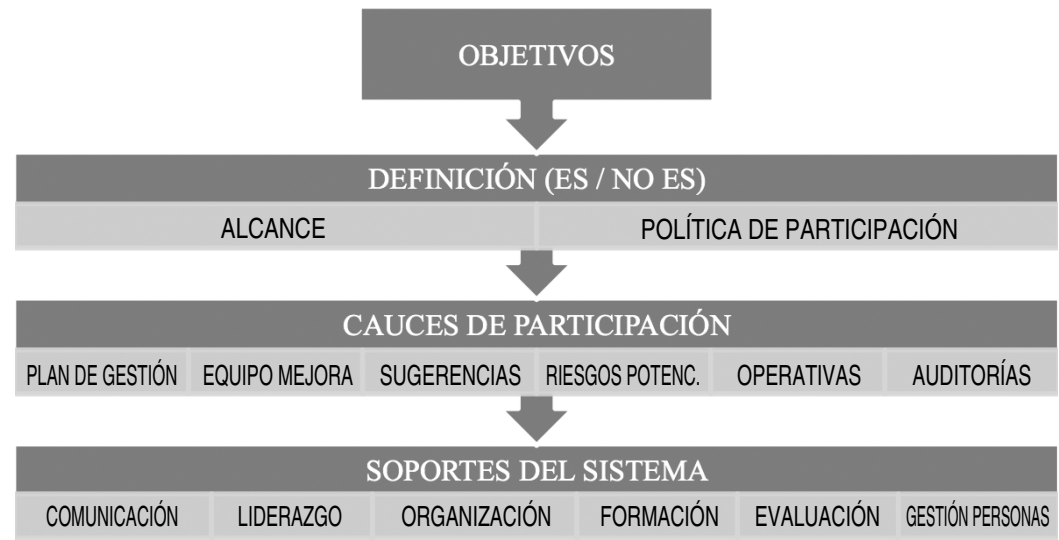

FUENTE: Fagor Ederlan.

El alcance de este Sistema de Participación es el siguiente: en la gestión de la mejora (contribuyendo al logro de los objetivos), en la gestión de los procesos (contribuyendo a satisfacer las necesidades de los clientes internos y externos de los procesos en los que se interviene) y en la gestión de las actividades (contribuyendo a la mejora, eficacia y seguridad de las tareas que se realizan). En cuanto a los mecanismos más relevantes de profundización de la participación de las personas, destacan:

Trabajo en equipo: en la filial se entiende el trabajo en equipo como parte de su cultura, siendo el mecanismo más relevante de participación sistemática en la gestión de la entidad. La mayoría de personas, sea cual sea su ámbito, desarrollan su actividad diaria en equipos de trabajo.

Formación y educación para las personas: se distinguen dos tipos de formación y educación para los miembros de la plantilla de Fagor Ederlan Tafalla. Por un lado, existe una formación cooperativa dirigida exclusivamente a los socios trabajadores, de la que se encargan los "Responsables en Formación Cooperativa". Estos programas de educación se centran en la transmisión de la identidad y cultura cooperativa, los valores más filosóficos y los principios del cooperativismo. Por otro lado, existe un amplio ámbito formativo dirigido a todos los trabajadores de la plantilla sobre cuestiones más técnicas y operativas relacionadas con el puesto de trabajo concreto. Esto se complementa con una organización que favorece la promoción interna, la versatilidad de los puestos de trabajo y la flexibilidad de las personas en las áreas productivas. 
Comunicación Interna: La comunicación interna es un factor fundamental para vehiculizar la expresión participativa de las personas. Por ello se establecen flujos de información en sentido ascendente, descendente y horizontal entre todas las personas de la organización. Se distinguen tres canales principales de comunicación interna: Canal Social (comunicación bidireccional entre Consejo Rector y socios); Canal Ejecutivo (comunicación bidireccional entre Consejo de Dirección y socios/trabajadores); Canal Directo (comunicación unidireccional vía intranet, tableros, hojas informativas... cuyo punto de partida puede ser cualquier ámbito de la organización empresarial).

Sistema de sugerencias: es la vía de canalización de las aportaciones individuales de los trabajadores a la mejora en la gestión diaria de la cooperativa, erigiéndose como una herramienta de participación para todos los miembros de la plantilla. El objetivo principal de dicho sistema es contribuir a las mejoras en los procesos y en los puestos de trabajo mediante la participación individual y posterior construcción colectiva.

Identificación de riesgos potenciales: Con una lógica similar al sistema de sugerencias, existe este modelo que permite la participación de los trabajadores en la identificación de riesgos laborales.

Todos estos mecanismos de participación se desarrollan en torno a un factor clave de distribución de la organización productiva: la descentralización operativa en las denominadas "mini-compañías". Éstas son formas organizativas diseñadas para las áreas productivas, con las que se quiere dar más autonomía a los trabajadores, siendo ellos quienes decidan y solucionen los problemas en el mismo ámbito en el que se generan. Es decir, se trata de establecer una horizontalidad participativa para que el problema y la solución no sigan un proceso de abajo a arriba hasta la gerencia, existiendo en estas unidades un soporte y apoyo de todos los ámbitos de la empresa (calidad, prevención de riesgos...). Por tanto, las "mini-compañías" permiten materializar la formación adquirida a través de la autogestión y potenciar el trabajo en equipo, erigiéndose como vehículos de los distintos mecanismos participativos. Su finalidad es incrementar la productividad mediante la implicación de las personas.

\subsection{Resultados del Proyecto de Cooperativización de Tafalla}

En primer lugar, todos los entrevistados coinciden en que la cooperativización de la filial, gracias a las fuertes inversiones realizadas ( 60 millones de euros en un periodo de grave crisis económica) en la mejora de los medios tecnológicos, procesos productivos, modelos organizativos y formación de las personas, ha permitido la supervivencia de la filial y su mejor posición competitiva en el mercado. Así mismo, el nuevo modelo cooperativo ha dotado de una seguridad laboral a los socios y trabajadores y, a su vez, ha permitido lograr una mayor flexibilidad y adaptabilidad tanto a los cambios necesarios a nivel interno como a los condicionados desde el exterior, cuestión fundamental considerando las características del sector de la automoción, tan dinámico y competitivo, y el contexto de crisis económica. 
En el mismo sentido se expresaban la mayoría de entrevistados. Como señalaba el Presidente de Fagor Ederlan Tafalla, "la actitud y compromiso de las personas ha propiciado que la filial cooperativa haya soportado mejor la crisis de cómo lo hubiera hecho bajo la fórmula anterior. Gracias al apoyo inter-cooperativo de la matriz y MCC, así como a la adopción de medidas solidarias como las bajadas salariales en lugar de despidos, podemos considerar que estamos enfrentando la crisis en mejores condiciones que otras empresas del sector."

En esta línea, tal y como se desprende de nuestras entrevistas y de las investigaciones internas de Fagor Ederlan, la cooperativización de la filial, así como la integración del modelo de gestión y en concreto del sistema de participación de Fagor Ederlan, han favorecido exponencialmente el control democrático y el carácter participativo de las personas en la filial de Tafalla. La evolución en la naturaleza del trabajo desde una lógica capitalista a una cooperativista ha fomentado la autogestión y una horizontalidad tanto en las relaciones laborales como en el organigrama empresarial. Esto se refleja en la mayor identificación e implicación de los socios con el proyecto al haber aumentado su motivación y compromiso con la cooperativa.

Tal y como señalaba el socio entrevistado (6), "la realidad social y laboral de socios y trabajadores ha cambiado drásticamente con el nuevo modelo cooperativo adoptado, y mientras antes se funcionaba bajo esquemas jerárquicos, ahora existe un alto grado de compromiso y participación de las personas en la empresa".

A pesar de la difícil situación de crisis atravesada desde 2008 (precisamente cuando la planta se transforma en filial), con pérdidas económicas, reducciones en los anticipos laborales, imposibilidad de obtener retornos cooperativos, necesidad de mayores aportaciones de los socios para ampliaciones de capital, etc., el proyecto cooperativo ha evolucionado positivamente hasta la actualidad. Entre 2008 y 2014 se ha pasado de algo más de 350 socios a 485 socios, la propiedad de la filial en manos de éstos ha pasado del $12 \%$ a casi el $20 \%$ y no se ha despedido a ningún trabajador asalariado fijo. En el año 2014 se han obtenido beneficios tras varios años de pérdidas continuadas, y para el año 2015 se ha obtenido un importante contrato con Volkswagen que asegura la supervivencia de la empresa a largo plazo y hace prever importantes ganancias. Por otro lado, la conversión de buena parte de los trabajadores de Tafalla en socios como resultado de la cooperativización, ha supuesto un impacto positivo a nivel global del Grupo Fagor Ederlan en términos del grado de control democrático, aspecto patente en el hecho de que entre 2007 y 2008 el ratio de empleo cooperativo del Grupo haya aumentado del $36 \%$ al $49 \%$ hasta situarse en el año 2014 en el $65 \%$.

Sin embargo, tanto de nuestra investigación como de los trabajos internos de Fagor Ederlan y MIK, se extrae que el proceso de cooperativización también trajo consigo ciertos conflictos entre el colectivo de socios y el colectivo de asalariados, así como entre el Consejo de Dirección y un sector del Comité de Empresa y Sindicatos, que hasta hoy todavía no han sido resueltos. Así mismo, respecto a la participación de los trabajadores asalariados, a pesar de que gracias a la cooperativización de la empresa disfrutan de un elevado grado de participación en la gestión que afecta a sus áreas de acti- 
vidad y puestos de trabajo, así como de cierta representatividad a través del Comité de Empresa y Sindicatos, no se ha profundizado significativamente en vías que fomenten su participación en la propiedad, órganos de gobierno y resultados de la filial.

El trabajador entrevistado (7) se pronunciaba al respecto señalando que "mientras a los trabajadores asalariados nos han exigido bajadas salariales equiparables a las de los socios, existe la sensación general de que nuestro colectivo no puede opinar ni participar en las decisiones importantes que afectan a nuestra empresa".

\subsection{Implantación del modelo cooperativo en el resto de filiales de Fagor Ederlan}

El Grupo Fagor Ederlan sigue disponiendo de una filial capitalista en España, Fagor Ederlan Borja S.A. (Zaragoza), y tres en el extranjero, Fagor Ederlan Brasileira (Brasil), Fagor Ederlan Slovensko (Eslovaquia) y Fagor Ederlan Auto-Parts Kunshan (China). Respecto a la filial zaragozana, desde Fagor Ederlan aluden principalmente a una cuestión clave que impide se pudiese llevar a cabo un hipotético proyecto de cooperativización: la futura viabilidad de la planta. Destacan que la planta de Borja es mono producto y mono cliente al contar sólo con General Motors como socio, lo que implica que exista una gran incertidumbre que imposibilita que, desde Fagor Ederlan, se quiera impulsar allí un proyecto de cooperativización por el gran esfuerzo que conlleva y el elevado riesgo que supone el posible cierre de la planta. Siendo éste el factor clave, también valoran otras dificultades u obstáculos como la distancia geográfica y cultural, o el hecho de que en Aragón exista otra legislación cooperativa.

Respecto a las filiales extranjeras, desde Fagor Ederlan señalan que si cuestiones como la distancia geográfica y cultural o los obstáculos legales ya suponían serias dificultades para desarrollar el proceso de cooperativización en Tafalla, esta problemática se multiplica exponencialmente en dichas filiales extranjeras. Esto se traduce en que no se haya planteado la posibilidad de desarrollar un proyecto similar al de Tafalla en sus filiales china y eslovaca. En cambio, en su filial brasileña se intentó desarrollar un proceso de cooperativización que, si bien no tuvo éxito finalmente, sobresale como el único ejemplo en el ámbito cooperativo vasco en el que se ha estudiado la posibilidad de transformar una filial extranjera en cooperativa. Se eligió Brasil debido a que allí existe una cultura cooperativa, aunque muy distinta a la de aquí, y, en cierta medida, las diferencias culturales son menores si comparamos quizá con el caso eslovaco o chino. Finalmente, no se llegó a profundizar demasiado en este proyecto porque desde el principio no se pudieron atisbar grandes opciones de éxito.

En esta línea se pronunciaba Mikel Uribetxeberria, Presidente del Grupo Fagor Ederlan, "en Brasil analizamos las posibilidades de transformar una planta en cooperativa, pero su realidad legal y legislativa actual no permite muchas alegrías. Además, allá entienden las cooperativas de un modo muy diferente, quizá las perciban de manera más negativa". 
A raíz de la experiencia brasileña, desde Fagor Ederlan se comienzan a plantear otras posibilidades para democratizar estas filiales extranjeras. El objetivo es impulsar un funcionamiento más democrático en esas filiales, principalmente a través de la participación de sus trabajadores, de manera que se adecuen y equiparen al modelo cooperativo de la matriz. Para ello se establecen una serie de indicadores o parámetros que permiten extrapolar y adaptar en estas filiales diversos elementos que les habilitan para aproximarse a una lógica funcional y organizativa similar a la de la matriz cooperativa. Por ejemplo, integrando en la filial aspectos del modelo de gestión de la matriz, otorgando a los trabajadores una participación en la propiedad y resultados de la filial, realizando programas formativos y educativos, favoreciendo la autonomía y autogestión de los trabajadores en su puesto de trabajo o estableciendo unas condiciones laborales superiores a las existentes en el entorno sectorial del territorio extranjero.

En palabras del Presidente, "el objetivo es extender este espíritu a las empresas vinculadas, aunque formalmente no sean cooperativas. Como mínimo, los modelos de gestión y la manera de funcionar tienen que ser homologables". Al respecto, señala, "hemos lanzado una directriz para todas las plantas del grupo: se trata de unos diez parámetros para comparar las cooperativas con las filiales. Hablamos de aspectos como la participación en el capital, la distribución de resultados, programas formativos para las personas o compartir modelos de gestión".

En cualquier caso, estas directrices no se fijan como objetivos específicos dirigidos a una transformación sustancial de las filiales extranjeras, sino que se utilizan más bien como referencias o indicadores que permiten comparar el funcionamiento de éstas respecto al de la matriz cooperativa. Por tanto, el impacto de estas políticas no se ha traducido en resultados relevantes que hayan potenciado una lógica cooperativa, especialmente en términos de participación de los trabajadores.

En el caso de Fagor Ederlan Brasileira destacan su balance social, que integra diversos indicadores de seguimiento anual en la filial, su modelo de gestión y políticas de seguridad del trabajador, su cercanía con el movimiento cooperativo local brasileño y su compromiso con el entorno próximo, por ejemplo, a través de las inversiones orientadas a minimizar el impacto medioambiental. Sin duda, estos aspectos sintonizan con una lógica cooperativa y se traducen en que ésta sea la filial extranjera donde mayor grado de implantación ha tenido el modelo cooperativo pero, a su vez, también advertimos importantes insuficiencias en lo relativo a la participación de los trabajadores en la propiedad, órganos de gobierno o resultados. Los casos de Fagor Ederlan Slovensko y Fagor Ederlan Auto-Parts Kunshan resultan más llamativos, especialmente este último donde, si bien es cierto que se ha desarrollado un modelo interesante de aprovechamiento de sinergias e inter-cooperación con otras empresas ubicadas en el Parque Industrial Kunshan, destacan por otra parte las graves carencias de sus trabajadores, quienes no participan en la propiedad, resultados y gestión, y ni tan siquiera están representados en estructuras sindicales o equivalentes, contando con unas condiciones socio-laborales similares a las de empresas multinacionales convencionales del entorno. Al respecto, debemos señalar que las dificultades para implantar el modelo cooperativo en la filial china son notables, siendo 
por ejemplo un factor clave la falta de espíritu cooperativo entre sus trabajadores, ya que la cultura empresarial y laboral impide integrar en ellos una lógica cooperativista (Errasti, 2013b).

Como señalaba el Directivo de Fagor Ederlan entrevistado (2), "en las filiales extranjeras, especialmente en la china y eslovaca, no se ha profundizado en la participación de los trabajadores en la gestión, propiedad y resultados de las filiales y no se ha avanzado sensiblemente hacia un esquema de funcionamiento cooperativo debido a las grandes dificultades legales y culturales que supondría".

\section{4.- Dificultades y soluciones para extender el modelo cooperativo a las filiales capitalistas}

A continuación analizamos las principales complejidades y dificultades para extender y reproducir el modelo cooperativo en las filiales capitalistas, las cuales pueden subyacer tras la divergencia entre el discurso teórico de regeneración instaurado en Mondragón y las prácticas y experiencias concretas adoptadas por sus cooperativas en esta dirección. Podemos partir tomando como referencia el trabajo de Flecha y Ngai (2014), en el que a partir de una revisión de la literatura previa y de los resultados de su investigación sobre cinco grandes cooperativas de Mondragón de distintos sectores, agrupa las dificultades para extender el modelo cooperativo hacia sus filiales en cuatro grandes barreras: económicas, legales, culturales y relacionadas con la inversión.

Dichas barreras son claramente identificables en la experiencia de Fagor Ederlan. Las barreras económicas hacen referencia a que, antes de abrirse a la posibilidad de que los trabajadores pasen a ser socios, la filial debe ser rentable económicamente. Esta barrera es evidente en el caso de Fagor Ederlan Borja, donde debido a la incertidumbre sobre la viabilidad económica futura de la planta, se descarta desarrollar un proyecto de cooperativización. Las barreras legales se refieren fundamentalmente a que existen muchos países extranjeros que no cuentan con la legislación cooperativa pertinente 0 con una similar a la de País Vasco. Esta problemática se hizo evidente en el caso de Fagor Ederlan Brasil, donde el proyecto de cooperativización planteado inicialmente tuvo que ser retirado. Las barreras culturales se refieren a la falta de espíritu y cultura cooperativa entre los trabajadores de la filial. Esta cuestión se manifiesta claramente en el caso de las filiales china o eslovaca de Fagor Ederlan, donde la distinta cultura social y laboral dificulta la implementación de una lógica cooperativista en los trabajadores. Por último, la cuarta barrera está relacionada con el control de las inversiones, refiriéndose a que entre algunos socios de las matrices existe miedo a perder el control sobre el capital que invirtieron si trabajadores de filiales extranjeras pasan a ser socios, debido a su posible falta de compromiso con la cooperativa. Esta preocupación la encontramos igualmente, de una forma u otra, entre los socios entrevistados. 
Como señalaba el socio entrevistado (6), "difícilmente podemos asegurarnos de que los trabajadores de la filial china desarrollen un firme compromiso con una empresa extranjera que además funciona bajo un modelo cooperativo muy alejado de su cultura laboral y empresarial'.

Estos elementos son algunas de las bases del axioma que asume en su discurso la propia Mondragón para justificar que sus filiales extranjeras se constituyan como sociedades anónimas y no como cooperativas y, a partir de los cuáles, surge la propuesta desde la Corporación de adoptar fórmulas intermedias cimentadas en el fomento de la gestión participativa de los trabajadores en dichas filiales extranjeras.

Sin embargo, desde nuestro punto de vista, entendemos que la lógica del proceso de cooperativización de Fagor Ederlan Tafalla nos da ciertas claves extrapolables al ámbito de las filiales extranjeras para poder salvar este tipo de obstáculos. En este sentido, la transformación de la filial extranjera en una cooperativa mixta de trabajo asociado, participada por sus socios trabajadores y por la matriz, podría ser una buena fórmula. La idea es que, inicialmente, los socios trabajadores se hagan con un reducido porcentaje de la propiedad de su filial, y la parte restante mayoritaria quede en manos de la matriz. En primer lugar porque el desembolso monetario que exigiría para los nuevos socios hacerse con la propiedad total de la filial podría ser inalcanzable pero, igualmente, porque permite que al principio, cuando puede existir una mayor incertidumbre y desconfianza, la matriz pueda ejercer un control sobre la gestión de la filial. Obviamente, conforme los socios trabajadores de la filial adquieran una mayor experiencia y madurez cooperativa, la matriz debe ir cediendo su parte en la propiedad de la filial a sus socios, otorgándoles una mayor autonomía y capacidad de autogestión. Así mismo, es imprescindible que este proceso de cooperativización se sustente sobre unas bases previas de formación y educación cooperativa a los trabajadores de la filial con el objetivo de infundirles un espíritu y cultura cooperativista, y un compromiso con la empresa, necesarios para que en un futuro puedan gestionar con plena autonomía el funcionamiento de la filial bajo una lógica democrática y participativa.

Por otra parte, la cuestión legal sí que puede ser un obstáculo más complejo de salvar. Por ejemplo, en el caso de Fagor Ederlan Tafalla, este punto se pudo solventar gracias a la influencia de Mondragón, que posibilitó un cambio en la legislación navarra de cooperativas. Sin duda, parece mucho más complicado que esto se lleve a cabo en una filial extranjera, al menos, fuera de la Unión Europea, ya que en este ámbito sí existe la figura de la "Sociedad Cooperativa Europea"8. En este marco, sí que podríamos adscribirnos a la lectura que realiza la Corporación Mondragón y asumir la imposibilidad de constituir o transformar jurídicamente las filiales extranjeras en cooperativas, surgiendo la necesidad que ellos mismos plantean de adoptar pasos intermedios para democratizar esas filiales. En este marco, entendemos necesario profundizar en la participación de los trabajadores en los órganos de gobierno, mediante su mayor representatividad en los Consejos de Administración de las filiales y Consejos Rectores de las matrices o el mayor peso de Sindicatos y Comités de Empresa; en la participación de los trabajadores en la gestión, mediante un modelo de gestión en que la Dirección cuente obliga-

8.- Reglamento (CE) $n^{\circ}$ 1435/2003 de 22 de julio de 2003, del Consejo, relativo al estatuto de la sociedad cooperativa europea. 
toriamente con el criterio de los trabajadores para tomar una decisión (codecisión) o que directamente permita que ellos mismos tomen las decisiones (autogestión); en la participación de los trabajadores en la propiedad, a través de su progresiva entrada en el accionariado; y en la participación de los trabajadores en los resultados, mediante buenas políticas de distribución de resultados que consideren el reparto de beneficios entre trabajadores (Errasti, 2004).

En cualquier caso, como venimos constatando a lo largo de este estudio, las cooperativas de Mondragón, por lo general, no han avanzado significativamente en el desarrollo de políticas que potencien la participación de los trabajadores en sus filiales extranjeras y tampoco observamos que existan objetivos sólidos y concisos que vayan en esta línea, sino más bien procesos de reflexión que se materializan en indicadores de mínimos y referencias comparativas entre matrices y filiales extranjeras. En el caso de Fagor Ederlan, también se hace evidente que la política de parámetros comparativos propuesta para homologar el funcionamiento de sus filiales extranjeras al modelo cooperativo de la matriz, acorde al discurso de mínimos de otras cooperativas de su entorno y de la propia MCC, no ha permitido avanzar significativamente en el fomento de la participación de los trabajadores de dichas filiales, tal y como demuestran especialmente los casos de sus filiales eslovaca y china.

Esto nos lleva a deducir, tal y como aventurábamos anteriormente, que existen otros obstáculos o reticencias a nivel interno de las matrices y sus socios, que impiden se fomente la democracia y participación en las filiales extranjeras. En este sentido, entendemos que son clave factores como la acumulación y localización del poder en la toma de decisiones, y la retención de actividades y recursos de alto valor añadido en las matrices. Como sabemos, las cooperativas de Mondragón se internacionalizan para potenciar su competitividad y mantener así el empleo cooperativo en las matrices, y lo hacen desplazando actividades que ya no son rentables hacia las filiales extranjeras, reteniendo por tanto elementos estratégicos como procesos tecnológicamente avanzados, capacidad de I+D e inversión, actividades y productos de mayor valor añadido, etc. Esto provoca que las filiales extranjeras deban desarrollarse bajo estrechas relaciones de dependencia, lo que permite su control por parte de las matrices que implica que la toma de decisiones relevantes se concentre en las matrices. Así, en tanto que esa filial se transforme en cooperativa y pase su propiedad, gestión y resultados a manos de sus socios 0 , aun en menor grado, se potencie la participación de sus trabajadores, ese control por parte de la matriz puede verse cuestionado ya que, en una relación matriz-filial más equivalente, la filial podrá exigir una distribución más homogénea de recursos y actividades estratégicas, la homologación de condiciones socio-laborales, etc. En última instancia, esto podría generar cierta incertidumbre entre los socios de la matriz, quienes pueden ver peligrar sus puestos de trabajo.

Por tanto, en la línea que ya se presumía en investigaciones anteriores (Errasti, 2013a, 2013b), parece que existe una quinta barrera, probablemente la más relevante, referente a que no existe un interés real en potenciar procesos de democratización en las filiales capitalistas, debido a que esa pérdida de control sobre ellas puede poner en peligro los puestos de trabajo de los socios cooperativistas en las matrices vascas. Esta idea es consistente con la reciente investigación de Heras (2014), en la que a través de diversas entrevistas con socios de las áreas de Finanzas, Industria y Distribución de MCC, se concluye que es la seguridad laboral el principal lazo con sus cooperativas. 


\section{5.- Conclusiones}

El contexto originado a partir de los años 90 en el marco de la globalización ha empujado a muchas de las cooperativas industriales de Mondragón a seguir procesos de internacionalización en aras de mantener su competitividad y salvaguardar el empleo cooperativo en las plantas vascas. La clave reside en cómo se han desarrollado éstos y a qué objetivos han atendido, evidenciándose que dichas cooperativas no han sido en general capaces de integrar el modelo cooperativo en sus dinámicas de expansión y crecimiento, especialmente si atendemos al funcionamiento de las filiales ubicadas en el extranjero. Ante este escenario parece que, en los últimos años, en el seno de MCC se está haciendo hincapié en la necesidad de una regeneración a través de la implantación de medidas que potencien la participación de los trabajadores en las filiales capitalistas. Sin embargo, como es también evidente en Fagor Ederlan, esta línea de discurso se está planteando sobre propuestas de mínimos y no sobre objetivos sólidos y concretos, por lo que no se está avanzando en la transformación sustancial de dichas filiales.

Como revela nuestro estudio de caso, existen factores, como la falta de cultura cooperativa entre los trabajadores de las filiales o los obstáculos legales, que dificultan la implantación del modelo cooperativo en las filiales capitalistas, pero parecen todavía más determinantes otros obstáculos o reticencias existentes a nivel interno de las matrices, como la concentración del poder de decisión o la retención de las actividades y recursos estratégicos para mantener el empleo, que obstaculizan incluso la potenciación de la autonomía de estas filiales a través de la participación de sus trabajadores. Por tanto, si bien es cierto que Fagor Ederlan se erige como una de las entidades de MCC más comprometidas con la extensión del modelo cooperativo en sus filiales vinculadas, tal y como demuestran sus proyectos de cooperativización en Bergara, Usurbil y Tafalla, y ciertas buenas prácticas en su filial brasileña, debe tratar de consolidar y reproducir la lógica de estos proyectos cooperativos en el conjunto de su organización, extendiéndose también a sus filiales extranjeras a pesar de las dificultades que entraña. El proyecto de cooperativización de Tafalla nos ha dado claves que ponen de manifiesto las posibilidades para abordar este escenario, como la opción de que esas filiales se constituyan como cooperativas mixtas en las que su control por parte de la matriz vaya disminuyendo conforme los socios trabajadores adquirieran experiencia en la gestión democrática y eficiente de la filial, o la vital relevancia de implementar procesos previos de información, formación y educación cooperativa a los trabajadores.

El estudio del Grupo Fagor Ederlan nos ha proporcionado un marco real para analizar estas contradicciones, las dificultades existentes y las posibles soluciones que se pueden plantear para superarlas. Las limitaciones de nuestra investigación residen fundamentalmente en el hecho de que se analiza este único caso empresarial, y por tanto, a la hora de extraer conclusiones para el conjunto de 
las cooperativas multinacionales de MCC, debemos ser cautelosos. Sin embargo, es de destacar que los resultados de nuestras entrevistas están en la línea de los resultados de los trabajos internos desarrollados en Fagor Ederlan y en MIK, en los que se realizan un mayor número de entrevistas, y las conclusiones de nuestra investigación son consistentes con trabajos recientes similares sobre cooperativas de distintas áreas del Grupo Mondragón (Heras, 2014; Storey et al, 2014; Luzarraga e Irizar, 2012 o Flecha y Ngai, 2014).

Así mismo, nuestra investigación ha aportado un importante valor añadido a la literatura sobre el tema en tanto que la elección concreta de Fagor Ederlan ha contado con fuertes potencialidades para alcanzar satisfactoriamente nuestro objetivo de estudio. Por un lado, tras la caída de Fagor Electrodomésticos, Fagor Ederlan se sitúa como una de las cooperativas de mayor tamaño e importancia de la división industrial de Mondragón, así como uno de los buques insignia del Grupo. Por otro lado, el análisis del proceso de cooperativización desarrollado en su filial de mayor tamaño, Fagor Ederlan Tafalla, es uno de los proyectos de regeneración más potentes impulsados en el ámbito de Mondragón, y nos ha permitido identificar ciertas claves que pueden ser extrapolables a la transformación de las filiales extranjeras. Consideramos además que nuestra investigación puede tener un impacto interesante a nivel empresarial pudiendo fomentar el desarrollo de más procesos de cooperativización, ya que da a conocer los entresijos de un proyecto tan complejo, y además pionero, como es el de Fagor Ederlan Tafalla, el cual se erige como un ejemplo de transformación socio-económica que puede servir de referencia para otras cooperativas del entorno que disponen de filiales capitalistas. Así mismo, podemos considerar también un impacto a nivel institucional, en tanto que se demuestra la necesidad por parte de las Administraciones Públicas de adaptar las legislaciones sobre cooperativas a estas nuevas realidades y modelos de cooperativización. Finalmente, desde nuestro punto de vista, una línea interesante de investigación futura en el ámbito de estudio de las cooperativas de Mondragón, podría ir orientada a profundizar en una de las cuestiones clave que identificamos a raíz del presente artículo; cómo diseñar, implementar y evaluar políticas de educación y formación cooperativista para los trabajadores de las filiales extranjeras que permitan sentar las bases para desarrollar futuros procesos de cooperativización en ellas.

\section{6.- Referencias bibliográficas}

ACS, Z. \& PRESTON, L. (1997): "Small and Medium-Sized Enterprises, Technology, and Globalization: Introduction to a Special Issue on Small and Medium-Sized Enterprises in the Global Economy", Small Business Economics, 9, 1-6. 
ALTUNA, L. (2008): La Experiencia Cooperativa de Mondragón. Una síntesis general, LANKIMondragon Unibertsitatea, Eskoriatza.

ARANDO, S., FREUNDLICH, F., GAGO, M., JONES, D. \& KATO, T. (2010): "Assessing Mondragon: Stability \& Managed Change in the Face of Globalization", Working Paper Number 1003, William Davidson Institute.

AZKARRAGA, J. (2007): Mondragón ante la globalización. La cultura cooperativa ante el cambio de época, LANKI-Mondragon Unibertsitatea, Eskoriatza.

AZKARRAGA, J., CHENEY, G. \& UDAONDO, A. (2012): "Workers Participation in a Globalized Market: Reflections on and from Mondragon", en Atzeni, M. (Ed.) Alternative Work Organisations (pp. 76102), Palgrave Macmillan, New York.

BAKAIKOA, B., ERRASTI, A. \& BEGIRISTAIN, A. (2004): "Gobierno y democracia en los grupos empresariales cooperativos ante la globalización. El caso de Mondragón Corporación Cooperativa", CIRIEC-España, Revista de Economía Pública, Social y Cooperativa, 48, 53-77.

BAKAIKOA, B., ETXEZARRETA, E. \& MORANDEIRA, J. (2013): "La regeneración de las Entidades de Economía Social. Un reto en el País Vasco", REVESCO, Revista de Estudios Cooperativos, $112,151-75$.

BAREA, J., JULIÁ, J. \& MONZÓN, J.L. (1999): Grupos Empresariales de la Economía Social en España, CIRIEC-España, Valencia.

BASTERRETXEA, I. \& ALBIZU, E. (2009): "General Training and Employment Policy: Mondragon's Lessons in coping with crisis and saving employment". En: 2nd International CIRIEC Research Conference on the Social Economy, CIRIEC, Östersund (Sweden).

BATSTONE, E. (1983): "Organization and Orientation: A Life Cycle Model of French Cooperatives", Economic and Industrial Democracy, 4, 139-61.

BEN-NER, A. (1984): "On the Stability of the Cooperative Type of Organization", Journal of Comparative Economics, 8(3), 247-60.

BRADLEY, K. \& GELB, A. (1982): "The Replication and Sustainability of the Mondragon Experiment", British Journal of Industrial Relations, 20(1), 20-33.

CHAVES, R., FAJARDO, G. \& NAMORADO, R. (2003): "Integración empresarial cooperativa: posibilidades, ventajas e inconvenientes". En: /l Coloquio de cooperativismo y economía social, CIRIECEspaña, Valencia.

CHENEY, G. (1999): Values at Work: Employee Participation Meets Market Pressure at Mondragon, Cornell University Press, Ithaca-N.Y.

CLAMP, C. (2000): "The Internationalization of Mondragon", Annals of Public and Cooperative Economics, 71(4), 557-77. 
CORNFORTH, C. (1995): "Patterns of Cooperative Management: Beyond the Degeneration Thesis", Economic and Industrial Democracy, 16(4), 487-523.

CORNFORTH, C., THOMAS, A., LEWIS, R. \& SPEAR, R. (1988): Developing Successful Working CoOperatives, Sage Publications, London.

CÔTÉ, D. (2001): Holdings Coopératifs: Evolution ou Transformation définitive?, De Boeck, Brussels.

DEFOURNY, J. (1999): L'Économie Social au Nord et au Sud, Jalons De Boeck, Paris.

DIMAGGIO, P. \& POWEL, W. (1983): "The Iron Cage Revisited: Institutional Isomorphism and Collective Rationality in Organizational Fields", American Sociological Review, 48, 147-60.

ELLERMAN, D. (1984): "Entrepreneurship in the Mondragon Co-operatives", Review of Social Economy, Vol. XLII, 272-94.

ELORTZA, E., ALZOLA, I. \& LÓPEZ, U. (2012): "La gestión de la crisis en la Corporación Mondragón", Ekonomiaz, 79(1), 58-81.

ERRASTI, A. (2004): "Modelos de Empresa Multinacional Democrática o Cooperativa", Cuadernos de Gestión, 4(2), 3-29.

ERRASTI, A. (2013a): "Tensiones y Oportunidades en las Multinacionales Coopitalistas de Mondragón: El Caso de Fagor Electrodomésticos, Sdad. Coop.", REVESCO, Revista de Estudios Cooperativos, 113, 30-60.

ERRASTI, A. (2013b): "Mondragon's Chinese Subsidiaries: Coopitalist Multinationals in Practice", Economic and Industrial Democracy. DOI: 10.1177/0143831X13511503.

ERRASTI, A. \& MENDIZABAL, A. (2007): "The impact of globalization and relocation strategies in large co-operatives: the case of the Mondragon Co-operative Fagor Electrodomésticos S.Coop.", Advances in the Economic Analysis of Participatory and Labor-managed Firms, 10, 265-287.

ERRASTI, A., HERAS, I., BAKAIKOA, B. \& ELGOIBAR, P. (2003): "The Internationalisation of Cooperatives: The Case of the Mondragon Cooperative Corporation", Annals of Public and Cooperative Economics, 74(4), 553-84.

ESTRIN, S. \& JONES, D.C. (1992): "The Viability of Employee-Owned Firms: Evidence from France", Industrial and Labour Relations Review, 45(2), 323-38.

FLECHA, R. \& NGAI, P. (2014): "The challenge for Mondragon: Searching for the cooperative values in times of internationalization", Organization, 21(5), 666-82.

FRÖBEL, F., HEINRICHS, J. \& KREYE, O. (1980): The new international division of labour: Structural unemployment in industrialised countries and industrialisation in developing countries, Cambridge University Press, Cambridge. 
GEMSER, G., BRAND, M. \& SORGE, A. (2004): "Exploring the Internationalisation Process of Small Businesses: A Study of Dutch Old and New Economy Firms", Management International Review, $44(2), 127-50$.

HARVEY, D. (2005): A Brief History of Neoliberalism, Oxford University Press, Oxford-New York.

HEDLUND, G. \& KVERNELAND, A. (1985): "Are Strategies for Foreign Market Entry Changing? The Case of Swedish Investment in Japan", International Studies of Management and Organization, 15(2), 41-59.

HERAS, I. (2014): "The ties that bind? Exploring the basic principles of worker-owned organizations in practice", Organization, 21(5), 645-65.

HUET, T. (2000): “Can MCC go global?", Peace Review, 12(2), 283-86.

IRIZAR, I. (2005): Empresa Cooperativa y Liderazgo, Mondragon Unibertsitatea, Oñati.

IRIZAR, I. \& MACLEOD, G. (2008): "Innovación Emprendedora en el Grupo Mondragón. El Caso de sus Centros Tecnológicos", CIRIEC-España, Revista de Economía Pública, Social y Cooperativa, 60, 41-72.

LARRAÑAGA, J. (2006): Dilema del Cooperativismo en la Era de la Globalización, Federación de Cooperativas de Trabajo Asociado de Euskadi, Mondragón.

LUZARRAGA, J.M. (2008): Mondragon Multi-Localisation Strategy: Innovating a Human Centred Globalisation, PhD Thesis, University of Mondragon, Oñati.

LUZARRAGA, J.M. \& IRIZAR, I. (2012): "La Estrategia de Multilocalización Internacional de la Corporación Mondragón”, Ekonomiaz, 79, 114-145.

LUZARRAGA, J., ARANZADI, D. \& IRIZAR, I., (2007): "Understanding Mondragon globalization process: local job creation through multi-localization". En: ${ }^{\text {st }}$ CIRIEC International Research Conference on the Social Economy, Victoria (Canada).

MACLEOD, G. \& REED, D. (2009): "Mondragon's Response to the Challenges of Globalization: A Multilocalization Strategy". In: Reed, D. \& McMurtry, J. (Eds.), Co-operatives in a Global Economy: The Challenges of Co-operation across Borders, Cambridge Scholars Publishing, Newcastle, 111-40.

MASIÁ, C. (2011): "Fagor Ederlan vista desde la cocina. Entrevista con su Presidente Mikel Uribetxeberria", NEXE-Quaderns d'autogestió i economía cooperativa, 28, 51-53.

MCMURTRY, J.J. \& REED, D. (2009): Co-operatives in a Global Economy: The Challenges of Co-operation across Borders, Cambridge Scholars Press, Newcastle.

MEISTER, A. (1974): La Participation Dans Les Associations, Editions Ouvrières, Paris.

MEISTER, A. (1984): Participation, Associations, Development, and Change, Transaction Publishers, New Brunswick.

MENDIZABAL, A. (1998): La globalización, HIRU, Hondarribia. 
MENDIZABAL, A. (2011): "Globalización y Autogestión", REVES-Revista Vasca de Economía Social (GEZKI), 7, 7-28.

MENDIZABAL, A. \& ERRASTI, A. (2006): "Aspectos Económicos y Sociales de las Deslocalizaciones Productivas", Lan Harremanak Especial/Ale Berezia, 167-92.

MENDIZABAL, A., BEGIRISTAIN, A. \& ERRASTI, A (2005): "Deslocalizaciones y Empleo Cooperativo. El caso de Fagor Electrodomésticos, S. Coop.", CIRIEC-España, Revista de Economía Pública, Social y Cooperativa, 52, 237-66.

MIYAZAKI, H. (1984): "On Success and Dissolution of the Labour-Managed Firm in the Capitalist Economy", Journal of Political Economy, 92(5), 909-31.

MONDRAGÓN (2012a): Annual Report 2012, Mondragon Corporation, Eskoriatza.

MONDRAGÓN (2012b): Impacto de la internacionalización sobre la empresa doméstica. Mondragón Corporación, Eskoriatza.

MONDRAGÓN (2013): Modelo de Gestión Corporativo, Mondragón Corporación, Eskoriatza.

POTTER, B. (1891): The Cooperative Movement in Great Britain, Swan Sonnenschein \& Co, London.

RADRIGÁN, M. \& BARRÍA, C. (2007): El rol de las cooperativas en un mundo globalizado, IRECUS, Quebec.

REDONDO, G., SANTA CRUZ, I. \& ROTGER, J.M. (2011): "Why Mondragon? Analysing What Works in Overcoming Inequalities", Qualitative Inquiry, 17(3), $277-83$.

ROELANTS, B., DOVGAN, D., EUM, H. \& TERRASI, E. (2012): The Resilience of the Cooperative Model, CECOP-CICOPA, Brussels.

SPEAR, R. (2001): "Globalisation et stratégies des coopératives". In: Coté, D. (Ed.), Holdings Coopératifs: Evolution ou Transformation définitive?, De Boeck, Brussels, 115-38.

STOREY, J., BASTERRETXEA, I. \& SALAMAN, G. (2014): "Managing and resisting 'degeneration' in employee-owned businesses: A comparative study of two large retailers in Spain and the United Kingdom", Organization, 21(5), 626-44.

TULANKIDE (2004): "Empresa personalista, una opción más justa”, TuLankide, 493, 3-4.

URDANGARIN, C. (1999): "Internacionalización de Mondragón Corporación Cooperativa", Ekonomiaz, 44, 238-55.

WEBB, S. \& WEBB, B. (1920): A Constitution for the Socialist Commonwealth of Great Britain, Longman, London.

YIN, R.K. (2003): Case Study Research: Design and Methods, Sage Publications, London.

ZAMAGNI, S. \& ZAMAGNI, V. (2010): Cooperative Enterprise: Facing the Challenge of Globalization, Edward Elgar, Cheltenham. 